K. Shiraiwa

Nagoya Math. J.

Vol. 67 (1977), 121-138

\title{
A GENERALIZATION OF THE LEVINSON-MASSERA'S EQUALITIES
}

\author{
KENICHI SHIRAIWA
}

In his study of non-linear differential equations of the second order, $\mathrm{N}$. Levinson [3] defined the dissipative systems ( $D$-systems) which arise in many important cases in practice. To a dissipative system a transformation $T: \boldsymbol{R}^{2} \rightarrow \boldsymbol{R}^{2}$ called the Poincaré transformation is associated. Levinson used the Poincare transformation in the qualitative study of dissipative systems, and he [3] and Massera [5] obtained certain equalities between the number of subharmonic solutions of a dissipative systems under suitable conditions. We call these the Levinson-Massera's equalities.

In this paper we define a class of non-linear differential equations of order $n$ named $D^{\prime}$-systems, which is a subclass of $D$-systems for $n=2$ and still contains many important systems in practice. For a $D^{\prime}$ systems of order $n$, we associate a transformation $T: \boldsymbol{R}^{n} \rightarrow \boldsymbol{R}^{n}$, which we call the Poincaré transformation associated to the $D^{\prime}$-system. By a qualitative study of the Poincare transformation $T$, we obtain some equalities between the number of subharmonic solutions of a $D^{\prime}$-system under suitable conditions. These equalities coincide with the LevinsonMassera's equalities for $n=2$.

In the final section of this paper, we give an extension of the above results to a certain class of the time dependent vector fields on a compact differentiable manifold of arbitrary dimension.

\section{\$1. The Poincaré transformation}

Consider the following differential equation (1).

$$
\frac{d x}{d t}=f(t, x), \quad t \in \boldsymbol{R}, \quad x \in \boldsymbol{R}^{n}
$$

We assume the following condition (A) throughout the paper.

Received August 31, 1976. 
(A) (a) $f(t, x)$ is an $\boldsymbol{R}^{n}$-valued function of class $C^{1}$.

(b) $f(t, x)$ is periodic of period 1 with respect to the variable $t$. That is

$$
f(t+1, x)=f(t, x) \quad \text { for } t \in \boldsymbol{R}, \quad x \in \boldsymbol{R}^{n} .
$$

(c) There exists a solution $x=\varphi\left(t ; t_{0}, x_{0}\right)$ of the equation (1) defined on $-\infty<t<+\infty$ with any initial condition $\left(t_{0}, x_{0}\right) \in \boldsymbol{R} \times \boldsymbol{R}^{n}$.

By the assumption (A) the above solution $x=\varphi\left(t ; t_{0}, x_{0}\right)$ is unique with respect to the initial value $\left(t_{0}, x_{0}\right)$.

Now, a transformation $T: \boldsymbol{R}^{n} \rightarrow \boldsymbol{R}^{n}$ is defined by

$$
T(x)=\varphi(1 ; 0, x), \quad x \in \boldsymbol{R}^{n} .
$$

We call this $T$ the Poincaré transformation associated to the equation (1).

Proposition 1. $T$ is a diffeomorphism of class $C^{1}$ and is isotopic to the identity. Especially, $T$ is homotopic to the identity and orientation preserving.

Proof. It is easy to see that $T$ is a bijection with $T^{-1}(x)=\varphi(0 ; 1, x)$, $x \in \boldsymbol{R}^{n}$. Since $f(t, x)$ is of class $C^{1}, T$ and $T^{-1}$ are of class $C^{1}$ by the smooth dependence of the solutions of the differential equation with respect to the initial conditions. Therefore, $T$ is a diffeomorphism of class $C^{1}$.

Let $T_{t}: \boldsymbol{R}^{n} \rightarrow \boldsymbol{R}^{n}(0 \leqq t \leqq 1)$ be a map defined by $T_{t}(x)=\varphi(t ; 0, x)$, $x \in \boldsymbol{R}^{n}$. Then $T_{t}(0 \leqq t \leqq 1)$ is also a diffeomorphism of class $C^{1}$, and $T_{0}=1$, the identity map of $\boldsymbol{R}^{n}$, and $T_{1}=T$. Now, define a map $\tilde{T}: \boldsymbol{R}^{n}$ $\times[0,1] \rightarrow \boldsymbol{R}^{n}$ by $\tilde{T}(x, t)=T_{t}(x)=\varphi(t ; 0, x),(x, t) \in \boldsymbol{R}^{n} \times[0,1]$. Then $\tilde{T}^{.}$ is of class $C^{1}$ by the smooth dependence of the solutions with respect to the initial conditions. Therefore, $T$ is isotopic to the identity.

Since any diffeomorphism which is isotopic to the identity is homotopic to the identity and orientation preserving, the latter half of the proposition is proved.

Proposition 2. $\varphi(t+1 ; 0, x)=\varphi(t ; 0, T(x)), t \in \boldsymbol{R}, x \in \boldsymbol{R}^{n}$.

Proof. By our assumption (A) (b) it is easy to see that $\psi(t)$, $=\varphi(t+1 ; 0, x), t \in \boldsymbol{R}$ is a solution of (1) for any $x \in \boldsymbol{R}^{n}$. Since $\psi(0)$. $=\varphi(1 ; 0, x)=T(x), \psi(t)=\varphi(t ; 0, T(x))$ by the uniqueness of the solution of (1) with respect to the initial condition. 
The following corollaries are easy consequences of Proposition 2.

COROLLARY 1. For any integer $k$, the following equality holds.

$$
\varphi(t+k ; 0, x)=\varphi\left(t ; 0, T^{k}(x)\right), t \in \boldsymbol{R}, x \in \boldsymbol{R}^{n},
$$

where $T^{k}: \boldsymbol{R}^{n} \rightarrow \boldsymbol{R}^{n}$ is the $k$-fold iterate of $T$.

COROLLARY 2. Let $k$ be a positive integer. Then the following three conditions are equivalent.

(a) $x(t)$ is a periodic solution of (1) of period $k$.

(b) $x(0)$ is a fixed point of $T^{k}$.

(c) $x(0)$ is a periodic point of $T$ of period $k$.

Remark 1. If $x(t)$ is a periodic solution of rational period, then $x(0)$ is a periodic point of $T$. If $x(t)$ is a periodic solution of irrational period, then $x(0)$ is a recurrent point of $T$. Therefore, it is a nonwandering point of $T$.

From now on, we represent a point $x$ of $\boldsymbol{R}^{n}$ as a column vector $x=\left(\begin{array}{c}x_{1} \\ \vdots \\ x_{n}\end{array}\right)$, and we identify an $n \times m$ matrix $A$ with the linear map $\boldsymbol{R}^{n}$ $\rightarrow \boldsymbol{R}^{m}$ which assign $A x \in \boldsymbol{R}^{m}$ to each $x \in \boldsymbol{R}^{n}$.

Let $T: \boldsymbol{R}^{n} \rightarrow \boldsymbol{R}^{n}$ be a diffeomorphism of class $C^{1}$, and let $p \in \boldsymbol{R}^{n}$ be a fixed point of $T$. Then the derivative $D T(p): \boldsymbol{R}^{n} \rightarrow \boldsymbol{R}^{n}$ of $T$ at $p$ is a linear map corresponding to the Jacobian matrix of $T$ at $p$, and it is a non-singular $n \times n$ matrix.

Lemma 1. If $T: \boldsymbol{R}^{n} \rightarrow \boldsymbol{R}^{n}$ is a Poincaré transformation associated to a differential equation, then $\operatorname{det} D T^{k}(p)>0$ for any periodic point of $T$ of period $k$.

Proof. By Proposition 1, $T^{k}$ is orientation preserving for any positive integer $k$. Therefore, $\operatorname{det} D T^{k}(p)>0$ for any $p \in \boldsymbol{R}^{n}$ such that $T^{k}(p)=p$.

An isomorphism (or a non-singular square matrix) $L: \boldsymbol{R}^{n} \rightarrow \boldsymbol{R}^{n}$ is called hyperbolic if all the absolute values of its eigenvalues are different from 1.

Let $T: \boldsymbol{R}^{n} \rightarrow \boldsymbol{R}^{n}$ be a diffeomorphism of class $C^{1}$, and let $p \in \boldsymbol{R}^{n}$ be a fixed point of $T$. We call $p$ a hyperbolic fixed point of $T$, if $D T(p)$ is hyperbolic. 
Now, we shalligive:a criterion for a fixed point of a Poincaré transformation associated to a differential equation to be hyperbolic.

Let $T: \boldsymbol{R}^{n} \rightarrow \boldsymbol{R}^{n}$ be the Poincaré transformation associated to (1), and let $p \in \boldsymbol{R}^{n}$ be a fixed point of $T$. Then $x=\varphi(t ; 0, p)$ is a periodic solution of (1) of period 1 by Proposition 2. Now, consider the variation equation (3) of the equation (1) with respect to the periodic solution $x=\varphi(t ; 0, p)$ of period 1 .

$$
\frac{d x}{d t}=D_{x} f(t, \varphi(t ; 0, p)) x,
$$

where $D_{x} f(t, x)$ is the Jacobian matrix of $f(t, x)$ with respect to $x$. The variation equation (3) is a linear differetial equation with continuous periodic coefficients of period 1 .

Proposition 3. Let $W(t)$ be a fundamental matrix of the linear system (3). Then $D T(p)=W(1) W(0)^{-1}$.

Proof. Put $R(t)=D_{x} \varphi(t ; 0, p), t \in R$, where $D_{x} \varphi(t ; 0, x)$ is the Jacobian matrix of $\varphi(t ; 0, x)$ with respect to $x$. Then $R(t)$ is a matrix solution of (3) with the initial condition $R(0)=E_{n}$, the unit matrix.

Put $S(t)=W(t) W(0)^{-1}, t \in \boldsymbol{R}$. Then $S(t)$ is also a matrix solution of (3) with the initial condition $S(0)=E_{n}$. Therefore, $R(t)=S(t)$ for all $t$ by the uniqueness of the solution of (3) with respect to the initial condition. Especially $R(1)=S(1)$, that is $D T(p)=W(1) W(0)^{-1}$.

COROLlaRY. $p$ is a hyperbolic fixed point of $T$ if and only if all the real parts of the characteristic exponents of (3) are different from 0.

Proof. This follows from the definition of a hyperbolic fixed point and the Floquet theory using Proposition 3.

Let $T: \boldsymbol{R}^{n} \rightarrow \boldsymbol{R}^{\boldsymbol{n}}$ be a diffeomorphism of class $C^{1}$, and let $p \in \boldsymbol{R}^{n}$ be a hyperbolic fixed point of $T$. Now, let $E^{u}$ be the intersection of $\boldsymbol{R}^{n}$ and the direct sum of the generalized eigenspaces of $D T(p)$ corresponding to the eigenvalues $\lambda$ such that $|\lambda|>1$. Similarly, let $E^{s}$ be the intersection of $\boldsymbol{R}^{n}$ and the direct sum of the generalized eigenspaces of $D T(p)$ corresponding to the eigenvalues $\lambda$ such that $|\lambda|<1$.

Proposition 4. Under the above hypothesis and notations, the following properties hold.

(a) $\boldsymbol{R}^{n}=E^{u} \oplus E^{s}$ 
(b) $E^{u}$ and $E^{s}$ are $D T(p)$-invariant subspaces.

(c) Let $\lambda_{1}, \cdots, \lambda_{n}$ be the characteristic roots of $D T(p)$.

Then $\operatorname{dim} E^{u}=\sharp\left\{\lambda_{i} ;\left|\lambda_{i}\right|>1\right\}$ and $\operatorname{dim} E^{s}=\sharp\left\{\lambda_{i} ;\left|\lambda_{i}\right|<1\right\}$, where $\# M$ denotes the cardinarity of the set $M$.

(d) Let $\|\cdot\|$ be a norm of $\boldsymbol{R}^{n}$, and let $L=D T(p)$.

Then there exist constant $c(c>0)$ and $\lambda(0<\lambda<1)$ such that

$$
\begin{aligned}
& \left\|L^{-k}(x)\right\| \leqq c \lambda^{k}\|x\|, \quad x \in E^{u} \\
& \left\|L^{k}(x)\right\| \leqq c \lambda^{k}\|x\|, \quad x \in E^{s},
\end{aligned}
$$

where $k$ is any positive integer.

This is a standard results in Smale's theory on differentiable dynamical systems (cf. [8]).

Let $p$ be a hyperbolic fixed point of a diffeomorphism $T: \boldsymbol{R}^{n} \rightarrow \boldsymbol{R}^{n}$. Set

$$
\begin{aligned}
& W^{u}(p)=\left\{x \in \boldsymbol{R}^{n} ; \lim _{k \rightarrow \infty} T^{-k}(x)=p\right\} \text { and } \\
& W^{s}(p)=\left\{x \in \boldsymbol{R}^{n} ; \lim _{k \rightarrow \infty} T^{k}(x)=p\right\} .
\end{aligned}
$$

We call $W^{u}(p)$ (resp. $\left.W^{s}(p)\right)$ the unstable (resp. stable) manifold of $p$. The following theorem is a standard results in Smale's theory (Cf. [8]).

THEOREM. There exist suitable 1-1 immersions $\phi^{u}: E^{u} \rightarrow \boldsymbol{R}^{n}$ and $\phi^{s}: E^{s} \rightarrow \boldsymbol{R}^{n}$ such that

(a) $\phi^{u}\left(E^{u}\right)=W^{u}(p), \phi^{s}\left(E^{s}\right)=W^{s}(p)$,

(b) $\phi^{u}(0)=\phi^{s}(0)=p$, and

(c) $\operatorname{dim} W^{u}(p)=\operatorname{dim} E^{u}, \operatorname{dim} W^{s}(p)=\operatorname{dim} E^{s}$.

COROLlaRY. (d) $\operatorname{dim} W^{u}(p)$ is equal to the number of characteristic roots $\lambda$ of $D T(p)$ such that $|\lambda|>1$.

(e) $\operatorname{dim} W^{s}(p)$ is equal to the number of characteristic roots $\lambda$ of $D T(p)$ such that $|\lambda|<\mathbf{1}$.

This is an easy consequence of the above theorem and Proposition 4.

Let $p$ be a periodic point of $T$ of minimal period $n_{0}$. Then $p$ is a fixed point of $T^{n_{0}}$. If $D T^{n_{0}}(p)$ is hyperbolic, we call $\mathrm{p}$ a hyperbolic periodic point of $T$. Using $T^{n_{0}}$ instead of $T$, the similar theory can be developed as above for a periodic point of $T$. 


\section{$\S 2$. The fixed point index}

Let $T: \boldsymbol{R}^{n} \rightarrow \boldsymbol{R}^{n}$ be a continuous map, and let $p \in \boldsymbol{R}^{n}$ be an isolated fixed point of $T$. Define a map $1-T: R^{n} \rightarrow R^{n}$ by $(1-T)(x)=x-T(x)$, $x \in \boldsymbol{R}^{n}$. Then $(1-T)(p)=0$, and there exists a neighborhood $V$ of $p$ such that $(1-T)(V-\{p\}) \subset \boldsymbol{R}^{n}-\{0\}$ by our assumption. Therefore, $1-T$ induces a homomorphism

$$
(1-T)_{*}: H_{n}(V, V-\{p\}) \longrightarrow H_{n}\left(\boldsymbol{R}^{n}, \boldsymbol{R}^{n}-\{0\}\right),
$$

where $H_{n}(A, B)$ denotes the $n$-dimensional homology group of a pair $(A, B)$ with coefficients in the group of integers $Z$.

The groups $H_{n}(V, V-\{p\})$ and $H_{n}\left(\boldsymbol{R}^{n}, \boldsymbol{R}^{n}-\{0\}\right)$ are isomorphic to $\boldsymbol{Z}$, and if we fix an orientatation of $\boldsymbol{R}^{n}$, then there correspond unique generators $O_{V}$ and $O_{\boldsymbol{R}^{n}}$ of $H_{n}(V, V-\{p\})$ and $H_{n}\left(\boldsymbol{R}^{n}, \boldsymbol{R}^{n}-\{0\}\right)$ respectively. Using these generators $O_{V}$ and $O_{R^{n}}$, we get

$$
(1-T)_{*}\left(O_{V}\right)=m O_{R^{n}}
$$

for a suitable integer $m$. It is easily shown that $m$ does not depend on the choice of a neighborhood $V$ and an orientation of $\boldsymbol{R}^{n}$. The integer determined above is called the fixed point index of $T$ at $p$ and is denoted by $\operatorname{index}_{T}(p)$.

Proposition 5. Let $T: \boldsymbol{R}^{n} \rightarrow \boldsymbol{R}^{n}$ be a diffeomorphism of class $C^{1}$, and let $p \in \boldsymbol{R}^{n}$ be a hyperbolic fixed point of $T$. Then $p$ is an isolated fixed point, and the fixed point index is given as follows.

$$
\operatorname{index}_{T}(p)=\left\{\begin{aligned}
1 & \text { if } \operatorname{det}(1-D T(p))>0, \\
-1 & \text { if } \operatorname{det}(1-D T(p))<0,
\end{aligned}\right.
$$

where $\operatorname{det}(1-D T(p))$ is the determinant of the matrix $1-D T(p)$ and 1 is the unit matrix.

Proof. By a theorem of Hartman ([2], p. 245, Lemma 8.1), $T$ restricted on a suitable neighborhood of $p$ is topologically equivalent to $D T(p)$ restricted on some neighborhood of the origin 0 of $\boldsymbol{R}^{n}$. Therefore, it is sufficient to prove Proposition 5 in case that $T=D T(p)$ and $p=0$.

Now assume that $T$ is a hyperbolic linear isomorphism and $p=0$. Then $T=D T(p)$ and all the eigenvalues of $T$ are different from 1 . Therefore, $\operatorname{det}(1-T) \neq 0$. Thus, $0 \in \boldsymbol{R}^{n}$ is the only fixed point of $T$, and $p=0$ is an isolated fixed point of $T$. 
It is well known that a linear isomorphism $L: \boldsymbol{R}^{n} \rightarrow \boldsymbol{R}^{n}$ induces an isomorphism $L_{*}: H_{n}\left(\boldsymbol{R}^{n}, \boldsymbol{R}^{n}-\{0\}\right) \rightarrow H_{n}\left(\boldsymbol{R}^{n}, \boldsymbol{R}^{n}-\{0\}\right)$, and

$$
L_{*}\left(O_{R^{n}}\right)=\left\{\begin{aligned}
O_{R^{n}} & \text { if } \operatorname{det} L>0, \\
-O_{R^{n}} & \text { if } \operatorname{det} L<0,
\end{aligned}\right.
$$

Putting $L=1-T=1-D T(p)$, we obtain Proposition 5 .

Proposition 6. Let $T: \boldsymbol{R}^{n} \rightarrow \boldsymbol{R}^{n}$ be a diffeomorphism of class $C^{1}$, and let $p \in \boldsymbol{R}^{n}$ be a hyperbolic fixed point of $T$. Let $r$ be the number of the real characteristic roots $\lambda_{i}$ of $D T(p)$ such that $\lambda_{i}>1$. Then $\operatorname{index}_{T}(p)$ $=(-1)^{r}$.

Proof. Let $\lambda_{1}, \cdots, \lambda_{n}$ be the characteristic roots of $D T(p)$. Then $\operatorname{det}(1-D T(p))=\left(1-\lambda_{1}\right) \cdots\left(1-\lambda_{n}\right)$. If $\lambda_{i}$ is a complex number, there exists some $j$ such that $\lambda_{j}=\bar{\lambda}_{i}$. Therefore, it does not affect on the sign of $\operatorname{det}(1-D T(p))$. Now, it is clear that the sign of $\operatorname{det}(1-D T(p))$ is equal to that of $(-1)^{r}$. Now Proposition 6 follows from Proposition 5.

Proposition 7. Let $T: \boldsymbol{R}^{n} \rightarrow \boldsymbol{R}^{n}$ be a diffeomorphism of class $C^{1}$, and let $p \in \boldsymbol{R}^{n}$ be a hyperbolic fixed point of $T$. If we put $u=\operatorname{dim} E^{u}$ and $L_{u}=D T(p) \mid E^{u}: E^{u} \rightarrow E^{u}$, then the following properties hold for any positive integer $k$.

(a) If $\operatorname{det} L_{u}>0$, then $\operatorname{index}_{T^{k}}(p)=(-1)^{u}$.

(b) If $\operatorname{det} L_{u}<0$, then index $T_{T^{2 k-1}}(p)=(-1)^{u+1}$ and $\operatorname{index}_{T^{2 k}}(p)=(-1)^{u}$.

Proof. Let $\lambda_{1}, \cdots, \lambda_{n}$ be the characteristic roots of $D T(p)$. Then $\left|\lambda_{i}\right| \neq 1$ for any $i$ by our hypothesis. Since $D T^{k}(p)=(D T(p))^{k}$, the characteristic roots of $D T^{k}(p)$ are $\lambda_{1}^{k}, \cdots, \lambda_{n}^{k}$. Therefore, the absolute values of any eigenvalues of $D T^{k}(p)$ are different from 1 . Therefore, $p$ is a hyperbolic fixed point of $T^{k}$.

Now assume the following (4).

$$
\left\{\begin{array}{l}
\lambda_{i} \text { is real and } \lambda_{i}>1 \text { for } 1 \leqq i \leqq r \\
\lambda_{i} \text { is real and } \lambda_{i}<-1 \text { for } r+1 \leqq i \leqq r+s, \\
\lambda_{i} \text { is complex and }\left|\lambda_{i}\right|>1 \text { for } r+s+1 \leqq i \leqq r+s+t \text { and } \\
\left|\lambda_{i}\right|<1 \text { for } r+s+t+1 \leqq i<n
\end{array}\right.
$$

Then $t$ is even for complex characteristic roots appear in pair, and $u=r+s+t$ by Proposition 4(c). Since $\operatorname{det} L_{u}=\lambda_{1} \cdots \lambda_{r+s+t}$ and the product of complex characteristic roots $\lambda_{r+s+1}, \cdots, \lambda_{r+s+t}$ is positive, the 
sign of $\operatorname{det} L_{u}$ is equal to that of $(-1)^{s}$.

If $\operatorname{det} L_{u}>0$, then $s$ is even. Therefore, $(-1)^{u}=(-1)^{r+s+t}=(-1)^{r}$. Now, let $r(k)$ be the number of the real characteristic roots $\lambda_{i}^{k}$ of $D T^{k}(p)$ such that $\lambda_{i}^{k}>1$. Then by (4) and the fact that the complex characteristic roots appear in pair with their conjugates, we conclude that $r(k)$ $\equiv r \bmod 2$. Therefore, index $T^{k}(p)=(-1)^{r(k)}=(-1)^{r}=(-1)^{u}$ by Proposition 6 and the above stated facts. This proves (a).

If $\operatorname{det} L_{u}<0$, then $s$ is odd. Therefore, $(-1)^{u}=(-1)^{r+s+t}=(-1)^{r+1}$. And if $k$ is even, then $r(k) \equiv r+s \equiv r+1 \bmod 2$ as above. Thus, index $_{T^{k}}(p)=(-1)^{r(k)}=(-1)^{u}$ if $k$ is even. If $k$ is odd, then $r(k)$ $\equiv r \bmod 2$. Therefore, index $_{T^{k}}(p)=(-1)^{r(k)}=(-1)^{r}=(-1)^{u+1}$. This completes the proof.

EXAMPLE 1. Let $n=2$, and let $T: \boldsymbol{R}^{2} \rightarrow \boldsymbol{R}^{2}$ be a Poincaré transformation associated to a differential equation. Let $p \in \boldsymbol{R}^{2}$ be a hyperbolic fixed point of $T$, and let $\lambda_{1}, \lambda_{2}\left(\left|\lambda_{1}\right| \leqq\left|\lambda_{2}\right|\right)$ be the characteristic roots of $D T(p)$. Then the following four cases occur since $\operatorname{det} D T(p)>0$ by Lemma 1.

(i) The point $p$ is a completely unstable fixed point of $T$ if $1 \leqq\left|\lambda_{1}\right| \leqq\left|\lambda_{2}\right|$. In this case, index $T_{T^{k}}(p)=1$ for any positive integer $k$.

(ii) The point $p$ is a completely stable fixed point of $T$ if $\left|\lambda_{1}\right| \leqq\left|\lambda_{2}\right|$ $<1$. In this case, index $T^{k}(p)=1$ for any positive integer $k$.

(iii) The point $p$ is a directly unstable fixed point of $T$ if $0<\lambda_{1}$ $<1<\lambda_{2}$. In this case, $\operatorname{index}_{T^{k}}(p)=-1$ for any positive integer $k$.

(iv) The point $p$ is an inversely unstable fixed point if $\lambda_{2}<-1$ $<\lambda_{1}<0$. In this case, index $\operatorname{Ten}_{T^{2 k-1}}(p)=1$ and $\operatorname{index}_{T^{2 k}}(p)=-1$ for any positive integer $k$.

Similar statements hold for hyperbolic periodic points of $T$.

Definition 1. Let $T: \boldsymbol{R}^{n} \rightarrow \boldsymbol{R}^{n}$ be a diffeomorphism of class $C^{1}$, and let $p$ be a hyperbolic fixed point of $T$. Let $R^{n}=E^{u} \oplus E^{s}$ be the direct sum decomposition of $\boldsymbol{R}^{n}$ with respect to $L=D T(p)$ as in Proposition 4 , and let $L_{u}=D T(p) \mid E^{u}: E^{u} \rightarrow E^{u}$.

(i) If $\operatorname{dim} E^{u}$ is even and $\operatorname{det} L_{u}>0$, then we call $p$ a fixed point of type $P D$.

(ii) If $\operatorname{dim} E^{u}$ is odd and $\operatorname{det} L_{u}>0$, then we call $p$ a fixed point of type $N D$.

(iii) If $\operatorname{dim} E^{u}$ is even and $\operatorname{det} L_{u}<0$, then we call $p$ a fixed point of type $P I$. 
(iv) If $\operatorname{dim} E^{u}$ is odd and $\operatorname{det} L_{u}<0$, then we call $p$ a fixed point of type $N I$.

For a hyperbolic periodic point $p$, we define its type similarly.

Using the above terminology Proposition 7 is restated as follows.

Proposition 7'. Under the same assumption of Proposition 7, the following properties hold for any positive integer $k$.

(i) If $p$ is of type $P D$, then index $\operatorname{Tex}_{k}(p)=1$.

(ii) If $p$ is of type $N D$, then $\operatorname{index}_{T^{k}}(p)=-1$.

(iii) If $p$ is of type PI, then $\operatorname{index}_{T^{2 k-1}}(p)=-1$ and $\operatorname{index}_{T^{2 k}}(p)=1$.

(iv) If $p$ is of type NI, then $\operatorname{index}_{T^{2 k-1}}(p)=1$ and $\operatorname{index}_{T^{2 k}}(p)=-1$.

EXAmple 2. If $n=2$, case (iii) of Definition 1 does not occur for any Poincaré transformations associated to differential equations by Lemma 1.

If $p$ is a completely unstable or stable fixed (or periodic) point of $T$, then it is of type $P D$. If $p$ is a directly unstable fixed (or periodic) point of $T$, then it is of type $N D$. If $p$ is an inversely unstable fixed (or periodic) point of $T$, then it is of type $N I$.

Remark 2. The notions of the completely unstable, completely stable, directly unstable and inversely unstable fixed point classify the local topological types of the hyperbolic fixed point for $n=2$. But our types defined above do not classify the local topological types of the hyperbolic fixed points.

The local topological type of a hyperbolic fixed point is classified by the dimension of $E^{u}$ and the signs of $\operatorname{det} L_{u}$ and $\operatorname{det} L_{s}$.

If $T$ is a Poincaré transformation associated to a differential equation, then $\operatorname{det} D T(p)>0$ for any fixed point $p$ of $T$ by Lemma 1 . Since $\operatorname{det} D T(p)=\operatorname{det} L_{u} \cdot \operatorname{det} L_{s}$ for a hyperbolic fixed point $p$, the local topological type of a hyperbolic fixed point is classified by the dimension of $E^{u}$ and the sign of $L_{u}$.

\section{§3. Levinson-Massera's equalities}

The following theorem is well known.

THEOREM. (Poincaré-Hopf-Lefschetz) Let $T: \boldsymbol{R}^{n} \rightarrow \boldsymbol{R}^{n}$ be a continuons map such that all the fixed points of $T$ are isolated. Suppose that there exists a subset $K$ of $\boldsymbol{R}^{n}$ such that 
(i) $K$ is homeomorphic to a closed n-disk,

(ii) $T(K) \subset K$ and

(iii) all the fixed points of $T$ are contained in $K$.

Then the following equality holds.

$$
\sum_{T(p)=p} \operatorname{index}_{T}(p)=1
$$

For a proof, see A. Dold [1] for example.

As an easy application, we have the following proposition.

Proposition 8. Let $T: \boldsymbol{R}^{n} \rightarrow \boldsymbol{R}^{n}$ be a diffeomorphism of class $C^{1}$, and let $k$ be a positive integer. Suppose that all the periodic points of $T$ of period $k$ are hyperbolic. Further, assume that there exists a subset $K$ of $\boldsymbol{R}^{n}$ such that

(i) $K$ is homeomorphic to a closed $n$-disk,

(ii) $T^{k}(K) \subset K$ and

(iii) all the periodic points of $T$ of period $k$ are contained in $K$.

Then the following equality holds.

$$
\sum_{T^{k}(p)=p} \operatorname{index}_{T^{k}}(p)=1
$$

Now we shall state the main theorem of this paper.

THEOREM 1. Let $T: \boldsymbol{R}^{n} \rightarrow \boldsymbol{R}^{n}$ be a diffeomorphism of class $C^{1}$ such that every periodic point of $T$ is hyperbolic. Further, assume that there exists a subset $K$ of $\boldsymbol{R}^{n}$ such that

(i) $K$ is homeomorphic to a closed $n$-disk,

(ii) $T(K) \subset K$ and

(iii) every periodic point belongs to $K$.

For each positive integer $q$, let $P D(q)$ (resp. $N D(q), P I(q), N I(q)$ ) denote the number of the periodic points of $T$ of minimal period $q$ of type $P D$ (resp. ND, PI, NI), and let $N(q)$ be the number of periodic points of $T$ of minimal period $q$. Then the following equalities hold.

$$
\begin{array}{cc}
\text { (5) } & N(q)=P D(q)+N D(q)+P I(q)+N I(q) \quad \text { for any } q . \\
\text { (6) } & P D(1)+N I(1)=N D(1)+P I(1)+1 . \\
\text { ( 7 ) } & N(1)=2(N D(1)+P I(1))+1 . \\
\text { ( 8 ) } & P D(q)+N I(q)=N D(q)+P I(q) \quad \text { if } q \text { is odd and } q>1 .
\end{array}
$$


(9) $\quad N(q)=2(P D(q)+N I(q))=2(N D(q)+P I(q)) \quad$ if $q$ is odd and $q>1$.

(10) $P D(q)+N I(q)+2 P I(q / 2)=N D(q)+P I(q)+2 N I(q / 2)$ if $q$ is even .

(11) $N(q)=2(N D(q)+P I(q)+N I(q / 2)-P I(q / 2))$ if $q$ is even.

The following corollary is immediate from Theorem 1.

COROLLARY. Under the same assumption of Theorem 1, the following properties hold.

(i) $N(1)$ is odd.

(ii) If $q$ is odd and $q>1$, then $N(q)$ is divisible by $2 q$.

(iii) If $q$ is even and $P I(q / 2)=N I(q / 2)$, then $N(q)$ is divisible by 2q. Especially, if $q$ is even and $P I(q / 2)=N I(q / 2)=0$, then $N(q)$ is divisible by $2 q$.

Proof of Theorem 1. By the assumption of Theorem 1, all the hypothesis of Proposition 8 are satisfied for any positive integer $k$, and the equality (5) holds by the definition.

Putting $k=1$ in Proposition 8 , we obtain the following equality by Proposition $(7)^{\prime}$.

$$
P D(1)-N D(1)-P I(1)+N I(1)=1 .
$$

This proves (6), and (7) is an easy consequence of (5) and (6).

In order to prove (8) and (10), we write down the equality of Proposition 8 in terms of $P D(q), N D(q), P I(q)$ and $N I(q)$. For this purpose, the following lemma is useful.

LEMMA 2. Let $p$ be a periodic point of $T$ of minimal period $r$, and let $k$ be a positive integer. Then the following properties hold.

(a) If $p$ is of type $P D$, then $p$ is a periodic point of $T$ of period $k r$ of type $P D$ for any $k$.

(b) If $p$ is of type $N D$, then $p$ is a periodic point of $T$ of period $k r$ of type ND for any $k$.

(c) If $p$ is of type PI, then $p$ is a periodic point of $T$ of period $k r$ of type PI (resp. PD) for any odd $k$ (resp. even $k$ ).

(d) If $p$ is of type NI, then $p$ is a periodic point of $T$ of period $k r$ of type NI (resp. ND) for any odd $k$ (resp. even k).

Proof. Since $D T^{k r}(p)=\left(D T^{r}(p)\right)^{k}$ for each $k$, Lemma 1 is easily derived from the definition. 
Now we come back to the proof of Theorem 1 .

Let $q$ be any odd integer greater than 1 . Let $q=p_{1}^{\alpha_{1}} \cdots p_{m}^{\alpha_{m}}$, where $p_{1}, \cdots, p_{m}$ are odd primes and $\alpha_{1}, \cdots, \alpha_{m}$ are positive integers. Now, we shall prove (8) by induction on $s(q)=\alpha_{1}+\cdots+\alpha_{m}$.

If $s(q)=1$, then $q$ is an odd prime, and the number of the fixed points. of $T^{q}$ of type $P D$ (resp. $N D, P I, N I$ ) is equal to $P D(1)+P D(q)$ (resp. $N D(1)+N D(q), P I(1)+P I(q), N I(1)+N I(q))$ by Lemma 2 . Therefore, by Proposition 8 applied to case $k=q$, we obtain the following equality.

$$
\begin{aligned}
(P D(1) & +P D(q))-(N D(1)+N D(q))-(P I(1)+P I(q)) \\
& +(N D(1)+N D(q))=1 .
\end{aligned}
$$

Subtracting (12) from the above equality, we obtain (8).

Assume that (8) holds for odd integers $r$ with $s(r)<s(s>1)$. We shall prove (8) for odd $q$ such that $s(q)=s$.

By Lemma 2, the number of the fixed points of $T^{q}$ of type $P D$ (resp. $N D, P I, N I$ ) is equal to $\sum_{r \mid q} P D(r)$ (resp. $\sum_{r \mid q} N D(r), \sum_{r \mid q} P I(r)$, $\left.\sum_{r \mid q} N I(r)\right)$. Therefore, we have the following equality by Proposition 8 .

$$
\sum_{r \mid q} P D(r)-\sum_{r \mid q} N D(r)-\sum_{r \mid q} P I(r)+\sum_{r \mid q} N I(r)=1
$$

By our inductive assumption, we have the following equality

$$
P D(r)-N D(r)-P I(r)+N I(r)=0 \quad \text { if } r \mid q \text { and } 1<r<q .
$$

Therefore, from the above equalities and (6), we obtain (8). Thus, (8) is proved for any odd $q$ with $q>1$, and (9) is an easy consequence of (8) and (5).

Let $q$ be an even integer, and let $q=2^{\alpha_{0}} p_{1}^{\alpha_{1}} \cdots p_{m}^{\alpha_{m}}$, where $p_{1}, \cdots, p_{m}$ are odd primes and $\alpha_{0}, \alpha_{1}, \cdots, \alpha_{m}$ are positive integers. Now, we shall prove (10) by induction on $t(q)=\alpha_{0}+\alpha_{1}+\cdots+\alpha_{m}$.

If $t=1$, then $q=2$, and the number of the fixed point of $T^{q}$ of type $P D$ (resp. $N D, P I, N I)$ is equal to $P D(1)+P D(2)+P I(1)$ (resp. $N D(1)+N D(2)+N I(1), P I(2), N I(2))$ by Lemma 2 . Therefore, the following equality holds by Proposition 8.

$$
\begin{aligned}
& (P D(1)+P D(2)+P I(1))-(N D(1)+N D(2)+N I(1)) \\
& \quad-P I(2)+N I(2)=1
\end{aligned}
$$

Subtracting (12) from the above equality, we obtain (10) for $q=2$. 
Assume that (10) holds for even integers $r$ such that $t(r)<t(t>1)$. We shall prove (10) for even $q$ such that $t(q)=t$.

Let $A=\left\{2^{\beta_{0}} p_{1}^{\beta_{1}} \cdots p_{m}^{\beta_{m}} ; 1 \leqq \beta_{0} \leqq \alpha_{0}-1, \quad 0 \leqq \beta_{i} \leqq \alpha_{i}, \quad i=1, \cdots, m\right\}$ $B=\left\{p_{1}^{\beta_{1}} \cdots p_{m}^{\beta_{m}} ; 0 \leqq \beta_{i} \leqq \alpha_{i}, i=1, \cdots, m\right\}$ and $C=\left\{2^{\alpha_{0}} p_{1}^{\beta_{1}} \cdots p_{m}^{\beta_{m}} ; 0 \leqq \beta_{i} \leqq \alpha_{i}\right.$, $i=1, \cdots, m\}$. Then, the set $\{r ; r \mid q\}$ is a disjoint union of $\mathrm{A}, \mathrm{B}$ and $\mathrm{C}$.

By Lemma 2 the number of the fixed points of $T^{q}$ of type $P D$ (resp. $N D, P I, N I)$ is equal to $\sum_{r \mid q} P D(r)+\sum_{r \in A} P I(r)+\sum_{r \in B} P I(r)$ (resp. $\left.\sum_{r \mid q} N D(r)+\sum_{r \in A} N I(r)+\sum_{r \in B} N I(r), \quad \sum_{r \in C} P I(r), \sum_{r \in C} N I(r)\right)$. Therefore, the following equality holds by Proposition 8 .

$$
\begin{gathered}
\left(\sum_{r \mid q} P D(r)+\sum_{r \in A} P I(r)+\sum_{r \in B} P I(r)\right)-\left(\sum_{r \mid q} N D(r)+\sum_{r \in A} N I(r)+\sum_{r \in B} N I(r)\right) \\
-\sum_{r \in C} P I(r)+\sum_{r \in C} N I(r)=1
\end{gathered}
$$

This is rewritten as follows.

$$
\begin{aligned}
\sum_{r \in A}( & P D(r)-N D(r)+P I(r)-N I(r)) \\
& +\sum_{r \in B}(P D(r)-N D(r)+P I(r)-N I(r)) \\
& +\sum_{r \in C}(P D(r)-N D(r)-P I(r)+N I(r))=1
\end{aligned}
$$

By (6), (8) and the inductive hypothesis, we have the following equalities.

$$
\begin{gathered}
P D(r)-N D(r)=P I(r)-N I(r)-2(P I(r / 2)-N I(r / 2)) \\
\quad \text { for } r \in A \cup C, r \neq q . \\
P D(r)-N D(r)=P I(r)-N I(r) \quad \text { for } r \in B, r \neq 1 . \\
P D(1)-N D(1)=P I(1)-N I(1)+1 .
\end{gathered}
$$

Putting these equalities into (13), we obtain the following equality.

$$
\begin{aligned}
2 \sum_{R \in A}(P I(r)-N I(r))-2 \sum_{r \in A}(P I(r / 2)-N I(r / 2)) \\
\quad+2 \sum_{r \in B}(P I(r)-N I(r))-2 \sum_{\substack{r \in C \\
r \neq q}}(P I(r / 2)-N I(r / 2)) \\
\quad+P D(q)-N D(q)-P I(q)+N I(q)=0 .
\end{aligned}
$$

By simplifying the above equality, we obtain the following equality.

$$
2(P I(q / 2)-N I(q / 2))+P D(q)-N D(q)-P I(q)+N I(q)=0
$$

This proves (10), and (11) is an easy consequence of (5) and (10). 
DEFINITION 2. Consider the equation (1) satisfying the condition (A). The equation (1) is called a $D^{\prime}$-system if there exists a subset $K$ of $\boldsymbol{R}^{n}$ satisfying the following two conditions.

(i) $K$ is homeomorphic to a closed $n$-disk.

(ii) For any solution $x(t)$ of (1) there exists a suitable number $t_{0} \in \boldsymbol{R}$ such that $x\left(t_{0}\right) \in K$, and if $x\left(t_{1}\right) \in K$ for some $t_{1} \in \boldsymbol{R}$, then $x(t) \in K$ for any $t \geqq t_{1}$.

Levinson and Massera ([3], [5]) called the equation (1) satisfying the condition (A) a $D$-system if it satisfies the following condition.

(iii) There exist a positive number $r$ and a positive integer $N$ satisfying the following condition.

For any solution $x(t)$ of (1) there exists a suitable number $t_{0} \in \boldsymbol{R}$ such that $\left\|x\left(t_{0}\right)\right\| \leqq r$ and $\|x(t)\| \leqq r$ for $t \geqq t_{0}+N$.

Proposition 9. If $n=2, a D^{\prime}$-system is a $D$-system.

Proof. This is clear from the definitions.

EXAMPLE 3. (Duffing's Equation) In the equation

$$
\frac{d^{2} x}{d t^{2}}+f(x) \frac{d x}{d t}+g(x)=e(t)
$$

We assume the following four conditions.

(i) $f(x), g(x)$ and $e(t)$ are of class $C^{1}$.

(ii) $e(t)$ is periodic of period 1 .

(iii) There exists a positive constant $c$ such that $f(x) \geqq c$.

(iv) $g^{\prime}(x) \geqq 0$ and $\lim _{x \rightarrow \infty} g(x)>E, \lim _{x \rightarrow-\infty} g(x)<-E$, where $E$ $=\max |e(t)|$.

The equation (14) is equivalent to the following 2-dimensional system $(14)^{\prime}$.

$$
\left\{\begin{array}{l}
\frac{d x}{d t}=y \\
\frac{d y}{d t}=-f(x) y-g(x)+e(t)
\end{array}\right.
$$

The equation $(14)^{\prime}$ (or (14)) is a $D^{\prime}$-system (Cf. Loud [4], Shiraiwa [7]).

EXAMPLE 4. (Levinson-Langenhop-Opial) In the equation 


$$
\frac{d^{2} x}{d t^{2}}+f\left(x, \frac{d x}{d t}\right) \frac{d x}{d t}+g(x)=e(t),
$$

we assume the following five conditions.

(i) $f(x, v), g(x)$ and $e(t)$ are of class $C^{1}$.

(ii) $e(t)$ is periodic of period 1 .

(iii) There exist positive numbers $m$ and $a$ such that

$$
f(x, v) \geqq m \quad \text { for }|x| \geqq a,|v| \geqq a .
$$

(iv) There exists a positive constant $M$ such that

$$
f(x, v) \geqq-M .
$$

(v) $\quad \liminf _{x \rightarrow \infty} g(x)>M a+E$ and $\limsup _{x \rightarrow-\infty} g(x)<-(M a+E)$, where $E=\max |e(t)|$.

The equation (15) is equivalent to the following (15)'.

$$
\left\{\begin{array}{l}
\frac{d x}{d t}=y \\
\frac{d y}{d t}=-f(x, y)-g(x)+e(t)
\end{array}\right.
$$

The equation $(15)^{\prime}$ (or (15)) is a $D^{\prime}$-system (Cf. Opial [6]).

THEOREM 2. Let the equation (1) be a $D^{\prime}$-system, and let $T: \boldsymbol{R}^{n}$ $\rightarrow \boldsymbol{R}^{n}$ be the Poincaré transformation associated to the equation (1). Suppose that any periodic points of $T$ is hyperbolic. Then the equalities (5) (11) hold for the periodic points of $T$.

Proof. By the definition of $D^{\prime}$-system, the Poincaré transformation associated to a $D^{\prime}$-system satisfies the assumptions of Theorem 1 .

COROLlaRY 1. Under the same assumption of Theorem 2, Corollary of Theorem 1 holds for the Poincaré transformation $T: \boldsymbol{R}^{n} \rightarrow \boldsymbol{R}^{n}$ associated to a $D^{\prime}$-system (1).

COROLlaRY 2. (Levinson-Massera [3], [5]) Suppose that the equation (1) is a $D^{\prime}$-system and $n=2$. Let $T: \boldsymbol{R}^{2} \rightarrow \boldsymbol{R}^{2}$ be the Poincaré transformation associated to the equation (1). Assume that all the periodic points of $T$ are hyperbolic. We denote by $C(q)$ (resp. $D(q), I(q)$ ) the number of the completely unstable or stable (resp. directly unstable, inversely unstable) periodic points of $T$ of minimal period $q$ ( $q$ : a positive integer). Then, the following equalities hold. 
$C(1)+I(1)=D(1)+1$.

$C(q)+I(q)=D(q)$ if $q$ is odd and $q>1$.

$C(q)+I(q)=D(q)+2 I(q / 2)$ if $q$ is even.

If we denote by $N(q)$ the number of the periodic points of $T$ of minimal period $q$, then the following equalities hold.

$N(q)=C(q)+D(q)+I(q)$ for any $q$.

$N(1)=2 D(1)+1$.

$N(q)=2 D(q)$ if $q$ is odd and $q>1$.

$N(q)=2 D(q)+2 I(q / 2)$ if $q$ is even.

Proof. This follows easily from Theorem 2 and Example 2.

\section{§4. An extension of Theorem 1 and 2}

In this section we shall discuss an extension of Theorem 1 and 2 to the case where $\boldsymbol{R}^{n}$ is replaced by a compact differentiable manifold of class $C^{1}$.

Let $M$ be a compact differentiable $n$-dimensional manifold of class $C^{1}$, and let $f: M \rightarrow M$ be a diffeomorphism of class $C^{1}$. For a fixed (or periodic) point of $f$, we can define the notion of hyperbolicity using a coordinate neighborhood. Also, the fixed point index can be defined similarly (Cf. [9], [1]).

The following theorem is well known (Cf. [1]).

THEOREM. (Lefschetz) Let $f: M \rightarrow M$ be a continuous map such that all the fixed points of $f$ are isolated. Let $f_{* i}: H_{i}(M) \rightarrow H_{i}(M)$ be the induced homomorphism on the $i$-th homology group $H_{i}(M)$ with coefficients in $\boldsymbol{R}$. Put $L(f)=\sum_{i=0}^{n}(-1)^{i}$ Trace $f_{* i}$ (the Lefschetz number).

Then the following equality holds.

$$
\sum_{f(p)=p} \operatorname{index}_{f}(p)=L(f)
$$

COROLlaRY. In addition to the hypothesis of the above theorem, we assume that $f$ is homotopic to the identity. Set $\chi(M)=\sum_{i=0}^{n}(-1)^{i}$ $\operatorname{dim} H_{i}(M)$ (Euler characteristic of $M$ ), where $\operatorname{dim} H_{i}(M)$ is the dimension of $H_{i}(M)$ as a vector space over $\boldsymbol{R}$. Then,

$$
\sum_{f(p)=p} \operatorname{index}_{f}(p)=\chi(M) \text {. }
$$

Proof. If $f$ is homotopic to the identity, then $L(f)=\chi(M)$. 
THEOREM 3. Let $f: M \rightarrow M$ be a diffeomorphism of class $C^{1}$ such that all the periodic points of $f$ are hyperbolic. Further, we assume that $L(f)=L\left(f^{k}\right)$ for any positive integer $k$. Then the following equalities hold.

$P D(1)+N I(1)=N D(1)+P I(1)+L(f)$.

$P D(q)+N I(q)=N D(q)+P I(q)$ if $q$ is odd and $q>1$.

$P D(q)+N I(q)+2 P I(q / 2)=N D(q)+P I(q)+2 N I(q / 2)$ if $q$ is even.

In the above equalities, $P D(q)$ (resp. $N D(q), P I(q), N I(q)$ ) is the number of the periodic points of $f$ of minimal period $q$ of type $P D$ (resp. ND,PI,NI). And if we denote by $N(q)$ the number of periodic points of $f$ of minimal period $q$, then the following equalities hold.

$N(q)=P D(q)+N D(q)+P I(q)+N I(q)$

$N(1)=2(N D(1)+P I(1))+L(f)$

$N(q)=2(N D(q)+P I(q))$ if $q$ is odd and $q>1$.

$N(q)=2(N D(q)+P I(q)+N I(q / 2)-P I(q / 2))$ if $q$ is even.

Proof. Theorem 3 is proved similarly to Theorem 1.

Let $X_{t}, t \in R$ be a time dependent vector field of class $C^{1}$ on $M$. Assume that $X_{t}$ is periodic of period 1 with respect to the variable $t$. Then it is easy to see that there exists a unique solution $x=\varphi\left(t ; t_{0}, x\right)$ of $X_{t}$ defined on $-\infty<t<+\infty$ for any initial value $\left(t_{0}, x_{0}\right) \in \boldsymbol{R} \times M$.

Now define a transformation $f: M \rightarrow M$ by $f(x)=\varphi(1 ; 0, x), x \in M$. We call this $f$ the Poincaré transformation associated to the periodic time dependent system $X_{t}$.

As Proposition 1, we can prove that $f$ is a diffeomorphism of class $C^{1}$ and is isotopic to the identity. Therefore, $f$ is homotopic to the identity, and $f$ is orientation preserving if $M$ is oriented.

THEOREM 4. Let $X_{t}, t \in \boldsymbol{R}$ be a differentiable time dependent vector field of class $C^{1}$ on a compact differentiable manifold $M$ of dimension $n$. And assume that $X_{t}$ is periodic of period 1 with respect to $t$. Now, let $f: M \rightarrow M$ be the Poincaré transformation associated to $X_{t}$. Assume further that all the periodic points of $f$ are hyperboic. Then the following equalities hold.

$$
\begin{aligned}
& P D(1)+N I(1)=N D(1)+P I(1)+\chi(M) . \\
& P D(q)+N I(q)=N D(q)+P I(q) \text { if } q \text { is odd and } q>1 . \\
& P D(q)+N I(q)+2 P I(q / 2)=N D(q)+P I(q)+2 N I(q / 2) \text { if } q \text { is even. } \\
& N(q)=P D(q)+N D(q)+P I(q)+N I(q) \text { for any } q . \\
& N(1)=2(N D(1)+P I(1))+\chi(M) .
\end{aligned}
$$


$N(q)=2(N D(q)+P I(q))$ if $q$ is odd and $q>1$.

$N(q)=2(N D(q)+P I(q)+N I(q / 2)-P(q / 2))$ if $q$ is even.

Here $P D(q), N D(q), P I(q), N I(q), N(q)$ and $\chi(M)$ are defined as above.

Proof. Theorem 4 is proved from Theorem 3 and the fact that $L\left(f^{k}\right)=\chi(M)$ for any positive integer $k$ in our case since $f$ is homotopic to the identity.

\section{REFERENCES}

[1] A. Dold: Fixed Point Index and Fixed Point Theorem for Euclidean Neighborhood Retracts, Topology 4 (1965), 1-8.

[2] P. Hartman: Ordinary Differential Equations, John Wiley \& Sons (1964).

[3] N. Levinson: Transformation Theory of Non-linear Differential Equations of the Second Order, Ann. of Math. 45 (1944), 723-737. Corrections, ibid. 49 (1948), 738.

[4] W. S. Loud: Boundedness and Convergence of Solutions of $x^{\prime \prime}+c x^{\prime}+g(x)=e(t)$, Duke Math. J. 24 (1957), 63-72.

[5] J. L. Massera: The Number of Subharmonic Solutions of Non-Linear Differential Equations of the Second Order, Ann. of Math. 50 (1949), 118-126.

[6] Z. Opial: Démonstration d'un Théorème de N. Levinson et C. Langenhop, Ann. Polon. Math. 7 (1960), 241-246.

[7] K. Shiraiwa: Boundedness and Convergence of Solutions of Duffing's Equation, Nagoya Math. J., 66 (1977), 151-166.

[ 8 ] S. Smale: Stable Manifolds for Differential Equations and Diffeomorphisms, Ann. Scuola Norm Sup. Pisa (3) 17 (1963), 97-116.

[ 9 ] S. Smale: Differentiable Dynamical Systems, Bull. Amer. Math. Soc. 73 (1967), $747-817$.

Department of Mathematics

College of General Education

Nagoya University 